Article

\title{
Theoretical Model of Radial Scattering Velocity of Fragments of the Reactive Core PELE Projectile
}

\author{
Liangliang Ding ${ }^{1,2} \mathbb{D}$, Jingyuan Zhou ${ }^{1}$, Xianwen Ran ${ }^{1, * \mathbb{C}}$, Wenhui Tang ${ }^{1, * \mathbb{C}}$, Xiaoguang Xue ${ }^{1,2}$ \\ and Yuli Zhao ${ }^{2}$ \\ 1 College of Liberal Arts and Sciences, National University of Defense Technology, Changsha 410073, China; \\ dingliangliang14@nudt.edu.cn (L.D.); zhoujingyuan19@nudt.edu.cn (J.Z.); \\ xuexiaoguang@nudt.edu.cn (X.X.) \\ 2 Beijing Special Engineering and Design Institute, Beijing 100028, China; way9092@163.com \\ * Correspondence: ranxianwen@nudt.edu.cn (X.R.); tangwenhui@nudt.edu.cn (W.T.); \\ Tel.: +86-135-7415-1246 (X.R.); +86-181-7512-1477 (W.T.)
}

Received: 22 June 2020; Accepted: 13 July 2020; Published: 17 July 2020

check for updates

\begin{abstract}
PELE projectile is a new type of armor-piercing warhead and has a more obvious fragmentation effect, which solves the problem of insufficient after-effects of conventional armor-piercing projectiles. Reactive material is a new type of energetic material, which has some characteristics similar to the traditional explosives but has better mechanical properties. Reactive material is insensitive under normal conditions, and it can release huge energy under external impact loading. This paper hopes to study the application of reactive materials to the inner core of PELE projectiles to further improve the fragmentation effect of PELE projectiles. The fragmentation effect of PELE projectile is mainly reflected in the radial scattering velocity of fragments after it perforates the target plate. In this paper, three energy sources for the radial scattering of fragments were obtained by analyzing the penetration process of PELE projectile, that is, the axial kinetic energy of outer casing, the radial compression potential energy generated by the inner core to the outer casing, and the chemical energy released by the reactive core material. Based on the simplification and assumptions, the theoretical model of radial scattering velocity of fragments of the reactive core PELE projectile was established. In addition, numerical simulations were carried out to verify the theoretical model. The results show that the numerical simulation results are in good agreement with the theoretical calculation results, which indicates that the model established in this paper is scientific and reasonable. The reactive core PELE projectile has a more significant fragmentation effect, which further enhances the comprehensive damage power of traditional PELE projectile. The theoretical model established in this paper can quickly assess the power of reactive core PELE projectile's fragmentation effect, which can be used to provide guidance and reference for engineering application.
\end{abstract}

Keywords: PELE projectile; reactive material; PTFE/Al; fragmentation effect; radial scattering velocity of fragments

\section{Introduction}

PELE (Penetrator with Enhanced Lateral Efficiency) projectile is a new type of armor-piercing projectile, which transforms part of the axial kinetic energy into the radial kinetic energy by using the difference in material properties between the outer casing and inner core [1-5]. PELE projectile is mainly composed of high-density casing and low-density core, the outer casing is usually made of heavy metals such as steel or tungsten, while the inner core is made of inert materials such as plastic or aluminum with relatively weak penetration performance. This kind of ammunition has good penetration ability and 
fragmentation effect, its body does not contain high-energy explosives and is not equipped with fuzes. It mainly relies on the physical function to make projectile penetrate the target, then the casing is broken into fragments to damage subsequent targets, which can better solve the practical problem of insufficient effectiveness of the conventional armor-piercing projectiles.

Reactive material is a new kind of energetic material, which was first discovered in 1970s, and then widely studied and applied [6]. The types of reactive materials mainly include: thermite (e.g., $\mathrm{Al} / \mathrm{CuO}$ ), metal/polymer mixture (e.g., $\mathrm{Al} / \mathrm{PTFE}$ ), intermetallic compound (e.g., $\mathrm{Al} / \mathrm{Ni}$ ), metastable intermolecular compound (e.g., $\mathrm{Al} / \mathrm{MoO}_{3}$ ), etc. In recent years, metal/polymer mixture type reactive materials have been paid more and more attention, especially the reactive materials based on fluoropolymers and active metals, which show great advantages in the application of weapons and equipment. The metal/polymer mixture type reactive material is relatively insensitive under normal conditions, but under high strain rate loading or collision conditions, there will be violent chemical reactions and release a lot of chemical energy. It has been shown that some properties of the reactive materials are similar to those of traditional explosives, but they have better mechanical properties than explosives, and they are insensitive and high energy under external impact loading. Taking the PTFE/Al (73.5\%/26.5\%) reactive material as an example, its unit mass energy and unit volume energy can reach 3.5 and 5 times of TNT explosive respectively, and the chemical energy released by the reaction under impact loading can reach dozens of times of its own kinetic energy $[7,8]$. In order to be able to clarify the reaction mechanism of the reactive material to further enhance its damage effect, a lot of research has been carried out, mainly focusing on the following aspects: formulations, molding and sintering process, energy release characteristics, mechanical properties and so on. Ran [9] designed six different types of reactive material formulations, and through the mechanical properties and the energy release characteristic analysis, a set of formulations with the best energy release capacity was obtained. Vasant [10] proposed a new molding and sintering process (cold pressing/sintering process) of PTFE/Al reactive material in 2003 and applied for a patent. This improved process better solves the problem of insufficient strength when PTFE/Al reactive materials are used as structural parts. Ding [11] and Zhou [12] conducted a comprehensive analysis of the energy release characteristics, impact-induced characteristics and mechanical properties of PTFE/Al/CuO with the help of a self-designed energy release measuring device, drop-weight test system and dynamic SHPB test system. Thadhani $[13,14]$ divided the chemical reaction of the reactive material into two stages according to the different starting time of the reaction of reactive material under impact loading: shock-induced chemical reaction (SICR) and shock-assisted chemical reaction (SACR).

The whole penetration process of traditional PELE projectile belongs to pure physical process, and there is no additional energy input. However, when the reactive material is applied to the inner core of PELE projectile, the reactive material can react and release energy actively after the projectile perforates the target plate. It will make the casing rupture and generate more fragments with higher radial scattering velocity, and it may even ignite other targets behind the detonating target plate, thus causing physical and chemical double damage to the target. Therefore, this paper proposes the concept of reactive core PELE projectile for the first time, that is, replacing the traditional core material with reactive material, in order to further improve the after-effect power of PELE projectile.

The performance index of PELE projectile is mainly reflected in two aspects: penetration ability and fragmentation effect. For the reactive core PELE projectile proposed in this paper, its biggest characteristic is mainly reflected in its fragmentation effect. At present, the research on the fragmentation effect of PELE projectile mainly focuses on the radial scattering velocity of fragments. Paulus [3] established the theoretical model of the axial residual velocity of PELE projectile and the radial scattering velocity of fragments, according to the principle of energy conservation and the one-dimensional linear elastic wave theory. Based on the principle of energy conservation and shock wave theory, Verreault [15-17] constructed the theoretical model of radial scattering velocity of fragments of PELE projectile by considering the interaction between the outer casing and the inner core. Zhu et al. [18] carried out a large number of PELE projectile penetration experiments, and analyzed the generation 
mechanism of the PELE projectile lateral effect. Based on the elastic wave theory, flathead projectile penetration theory, and the D'Alembert principle, the theoretical model of radial scattering velocity of fragments was established. Du et al. [19] regarded the inner core material of PELE projectile as fluid when the projectile impacts the target, and believed that the radial scattering velocity of fragments was mainly produced by the interaction of the outer casing and the inner core. Based on the theory of elastic wave and the theorem of kinetic energy and momentum, the theoretical model of radial scattering velocity of fragments of PELE projectile was established. Fan et al. [20,21] analyzed the energy conversion process of PELE projectile in the penetration process and gave the determination method of each part of the energy. Based on the shock wave theory and the principle of energy conservation, the approximate calculation formula of the radial scattering velocity of fragments of PELE projectile was obtained.

To sum up, there are few reseraches about the reactive core PELE projectile. The idea of applying reactive materials to the inner core of PELE projectile is first proposed in this paper, which is an innovative work in the research process of PELE projectile. Compared with the traditional PELE projectile, the reactive core PELE projectile has a more excellent fragmentation effect. Therefore, it is very necessary to establish a theoretical model of radial scattering velocity of fragments of the reactive core PELE projectile. Since the radial scattering velocity of fragments after PELE projectile perforating the target plate is difficult to accurately measure by experiments, this paper hopes to verify the theoretical model by means of numerical simulation. Therefore, the research work on the reactive core PELE projectile in this paper has important academic significance and broad application prospects.

\section{Structural Simplification and Penetration Analysis of the Reactive Core PELE Projectile}

\subsection{Structural Simplification of the Reactive Core PELE Projectile}

The main structural components of PELE projectile include high-density outer casing and low-density inner core, and it also includes nose cap and driving band in the actual engineering design. Taking a certain type of PELE projectile as an example, the overall view of the projectile body and the sectional view of the warhead are shown in Figure 1.

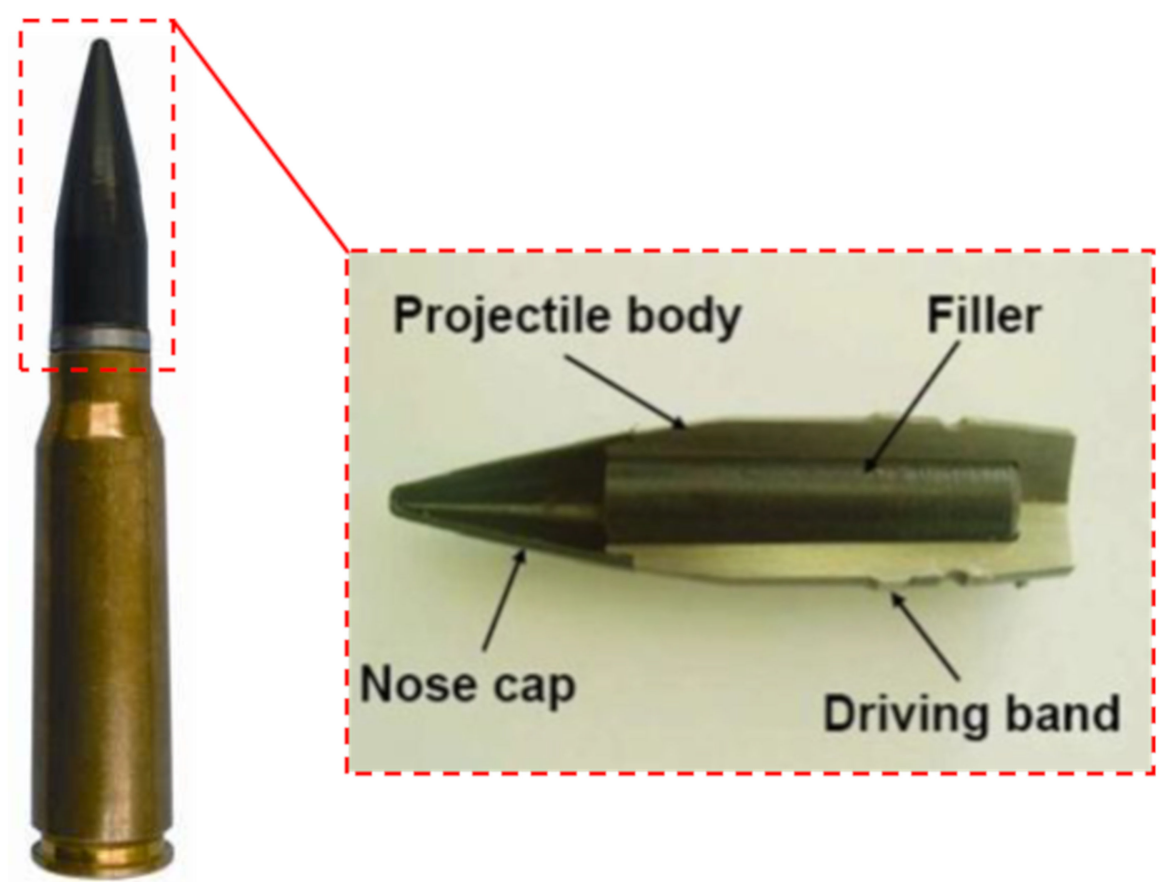

Figure 1. The overall view of PELE projectile body and the sectional view of the warhead. 
As can be seen from Figure 1, the structure of PELE projectile is relatively complex. In order to better conduct the following theoretical and numerical simulation studies, the structure of PELE projectile needs to be simplified. In the process of model simplification, the outer casing and inner core of the projectile body is retained, some minor components such as the nose cap and driving band are removed. Thus, the simplified structure diagram of the reactive core PELE projectile can be obtained as shown in Figure 2. The structure of the simplified reactive core PELE projectile is relatively simple, which provides convenience for the subsequent theoretical analysis and numerical simulation.

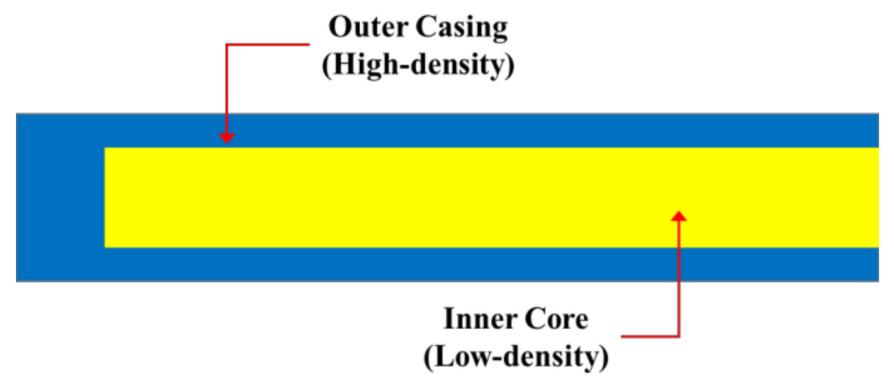

Figure 2. The overall view of PELE projectile body and the sectional view of the warhead.

\subsection{Description of Penetration Process of the Reactive Core PELE Projectile}

In this paper, the reactive core PELE projectile is mainly to replace the inner core of traditional PELE projectile from inert materials with PTFE/metal reactive materials, so it is necessary to clarify the basic reaction process of PTFE/metal reactive materials. Severe chemical reaction will occur between PTFE and metal powder under external loading, the whole chemical reaction process is shown in Figure 3 . The reaction process can be roughly divided into three stages as follows:

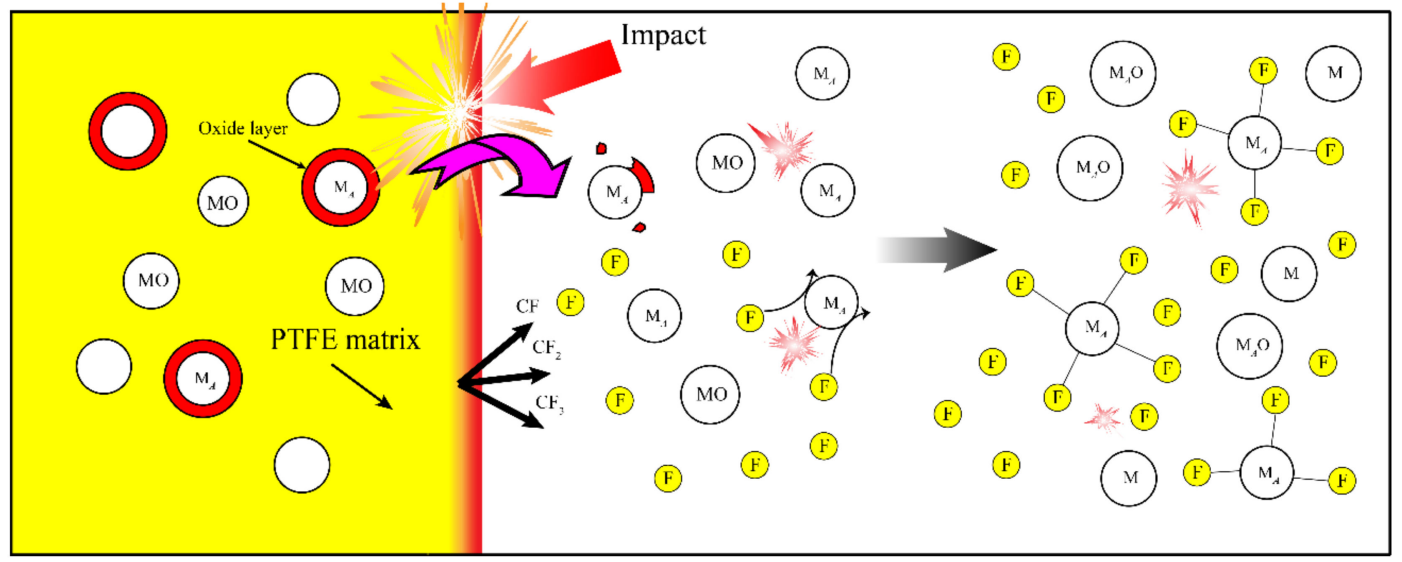

Figure 3. Schematic diagram of the reaction process between PTFE and active metals.

(1) Pre-reaction stage. This stage mainly includes the breakdown of oxide layer on the surface of metal particles and the thermal decomposition of PTFE molecules. Under the action of violent impact or high temperature, the metal oxide layer is broken and the metal particles are exposed to provide conditions for the subsequent redox reactions. Under violent impact or high temperature conditions, the $\mathrm{C}-\mathrm{C}$ bond of PTFE molecules will be broken and cracked into many $\mathrm{CF}_{\mathrm{x}}$ molecules with strong oxidation properties. The physical and chemical changes involved in the pre-reaction stage directly affect the ignition process of the entire reactive material. The ignition usually occurs at the interface between the metal particles and the fluoropolymer.

(2) Reaction stage. This stage mainly involves the redox reaction between metal particles and active molecule $\mathrm{CF}_{\mathrm{x}}$ formed by the thermal decomposition of PTFE. The entire reaction process is relatively complicated, involving reaction thermodynamics, reaction kinetics, etc. If the entire reaction 
is performed in an aerobic environment, oxygen molecules are also involved in the reaction of metal particles and thermal decomposition of PTFE molecules.

(3) Energy release stage. The intense redox reaction between metal particles and active molecule $\mathrm{CF}_{\mathrm{X}}$ can release a large amount of heat, which can continue to maintain the reaction of adjacent materials through heat conduction or shock wave propagation. A large amount of energy is released by the violent reaction of the active material, which in turn achieves work on the outside.

According to the analysis of the working principle of traditional PELE projectile, the typical simplified force state of traditional PELE projectile is shown in Figure 4.

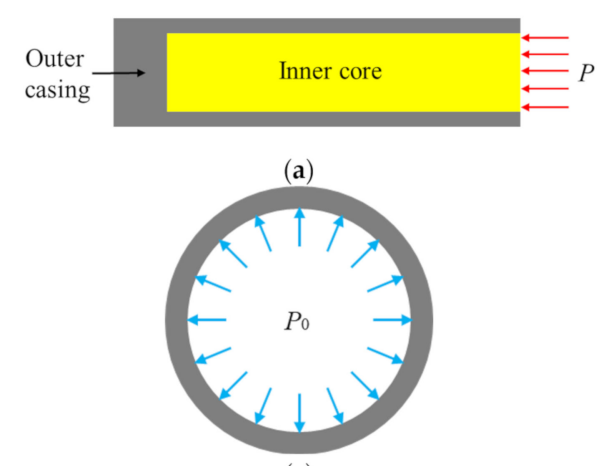

(c)
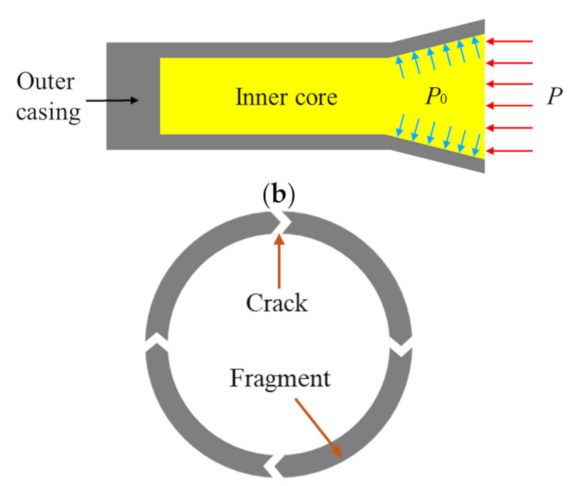

(d)

Figure 4. Typical simplified force state of the tarditional PELE projectile: (a) Force diagram of the projectile impacting the target at the initial moment; (b) Force diagram of the inner core when it is radially expanded; (c) Force diagram of the equivalent ring; (d) Simplified schematic diagram of the outer casing fracture.

For the reactive core PELE projectile, the reactive core materials show obvious inertia under low strain rate loading, but severe combustion and explosion effects will occur under high strain rate loading. Therefore, there is a certain difference between the penetration process of reactive core PELE projectile and that of traditional PELE projectile, which is mainly reflected in the energy source of outer casing breaking after perforating the target. The radial kinetic energy of outer casing after the reactive core PELE projectile perforates the target plate not only comes from the conversion of the axial compressive potential energy of the inner core material, but also includes the chemical energy released by the severe reaction of the inner core material. Therefore, from the perspective of energy conversion, it can be predicted that the radial scattering velocity of fragments corresponding to the reactive core PELE projectile is necessarily higher than that of the tarditional PELE projectile.

In order to facilitate the subsequent analysis, the penetration process of reactive core PELE projectile is divided into three stages, as shown in Figure 5. The three stages of penetration can be described as follows:

(a) Projectile initially penetrates the target. This stage means that the projectile has penetrated the target but has not perforated the target. At this stage, the inner core material is strongly compressed, and the density of inner core is in a stepped distribution, that is, the higher the density closer to the impact end, the lower the density closer to the projectile rear.

(b) Projectile partially perforates the target. At this stage, the impact end of projectile has completely perforated the target plate, but the projectile rear has not completely left the target plate, and part of the reactive inner core material has been activated to react. Under the combined action of the radial compression potential energy and the chemical energy generated by the reaction of the reactive inner core material, the outer casing breaks up and forms a large number of fragments.

(c) Projectile completely perforates the target. This stage means that the projectile has completely perforated and left the target plate. At this stage, the reactive inner core material further reacts and releases more energy, which results in the outer casing breaking into more fragments. 
It is worth noting that the reactive material is different from the traditional explosives, it belongs to the type of non-self-sustaining reaction, and not all reactions will occur under any conditions. Only when the pressure of the reactive material is higher than the critical pressure, can the violent combustion and explosion effect occur.

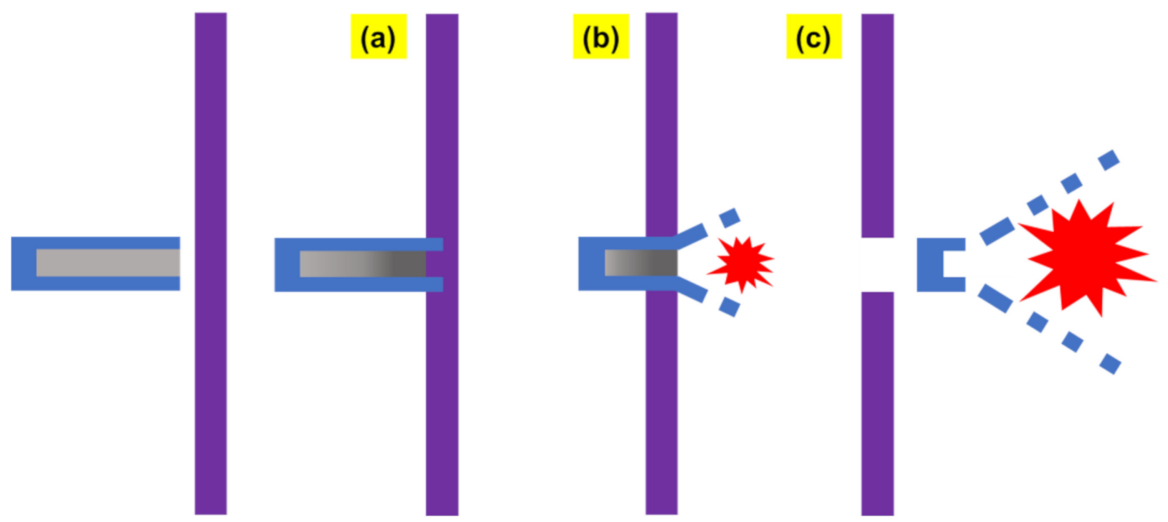

Figure 5. Schematic diagram of the penetration process of the reactive core PELE projectile. (a) Projectile initially penetrates the target; (b) Projectile partially perforates the target; (c) Projectile completely perforates the target.

\section{Theoretical Model of Radial Scattering Velocity of Fragments of Reactive Core PELE Projectile}

In the process of PELE projectile penetrating the target plate, the propagation of stress waves in the projectile is very complicated, and the outer casing is similar to the elastic waveguide in the penetration process. Because there are two interfaces inside and outside the casing, a certain dispersion phenomenon will occur when the stress wave propagates in the casing. The inner core material is not only subjected to the compression from the axial direction, but also subjected to the binding force of the casing. Therefore, it can be seen that in the whole penetration process of the projectile, the interaction among the outer casing, inner core and the target plate is relatively complex, and it will be accompanied by a variety of physical phenomena, mainly including: elastic wave propagation, plastic wave propagation, deformation and crushing of materials, etc. The velocity ranges of the reactive core PELE projectile examined in this paper is $600 \mathrm{~m} / \mathrm{s} \sim 1500 \mathrm{~m} / \mathrm{s}$. In order to obtain the radial scattering velocity of fragments generated by the reactive core PELE projectile when it penetrates the target plate, the following assumptions need to be made:

(a) Ignoring the mass loss of outer casing in the penetration process, the state of the inner core material is approximately regarded as fluid, and the outer casing material is regarded as the ideal elastic-plastic material.

(b) The force direction of the inner core to the outer casing is the outer normal direction of casing, and the friction between the inner core and the inner wall of casing is not considered when the inner core is compressed.

(c) Ignoring the energy dissipation caused by the deformation and breakage of outer casing, and it is considered that the compression potential energy stored in the inner core material due to Poisson effect and the chemical energy generated in the reaction are all released after perforating the target plate, which are all used to convert into the radial kinetic energy of fragments.

By analyzing the penetration process of the reactive core PELE projectile, the energy source of the radial scattering velocity of fragments can be summarized into the following three parts: the axial kinetic energy of outer casing which corresponds to the radial scattering velocity component $u_{r 1}$, the radial compression potential energy generated by the inner core to the outer casing which corresponds to the radial scattering velocity component of fragments $u_{r 2}$, and the chemical energy released by the 
reactive core material which corresponds the radial scattering velocity component of fragments $u_{r 3}$. The following analysis and research are based on these three energy sources.

In order to facilitate the following theoretical analysis, the structural parameters of PELE projectile and target plate are defined as follows: the initial velocity of PELE projectile is $u_{0}$, the length of outer casing is $L$, the length of inner core is $l$, the outer diameter of casing is $D$, the inner diameter of casing is $d$, the thickness of casing is $\delta$, the length-to-diameter ratio of casing is $\zeta=l / D$, the ratio of the inside and outside diameter of casing is $\gamma=d / D$, the thickness of target plate is $h$, the width of target plate is $\psi$, the material density is $\rho$, the material Poisson's ratio is $\mu$, the material elastic modulus is $E$, the subscript $t$ represents the target plate, the subscript $j$ represents the outer casing, and the subscript $f$ represents the inner core. The structural diagram of the PELE projectile and target plate is shown in Figure 6.
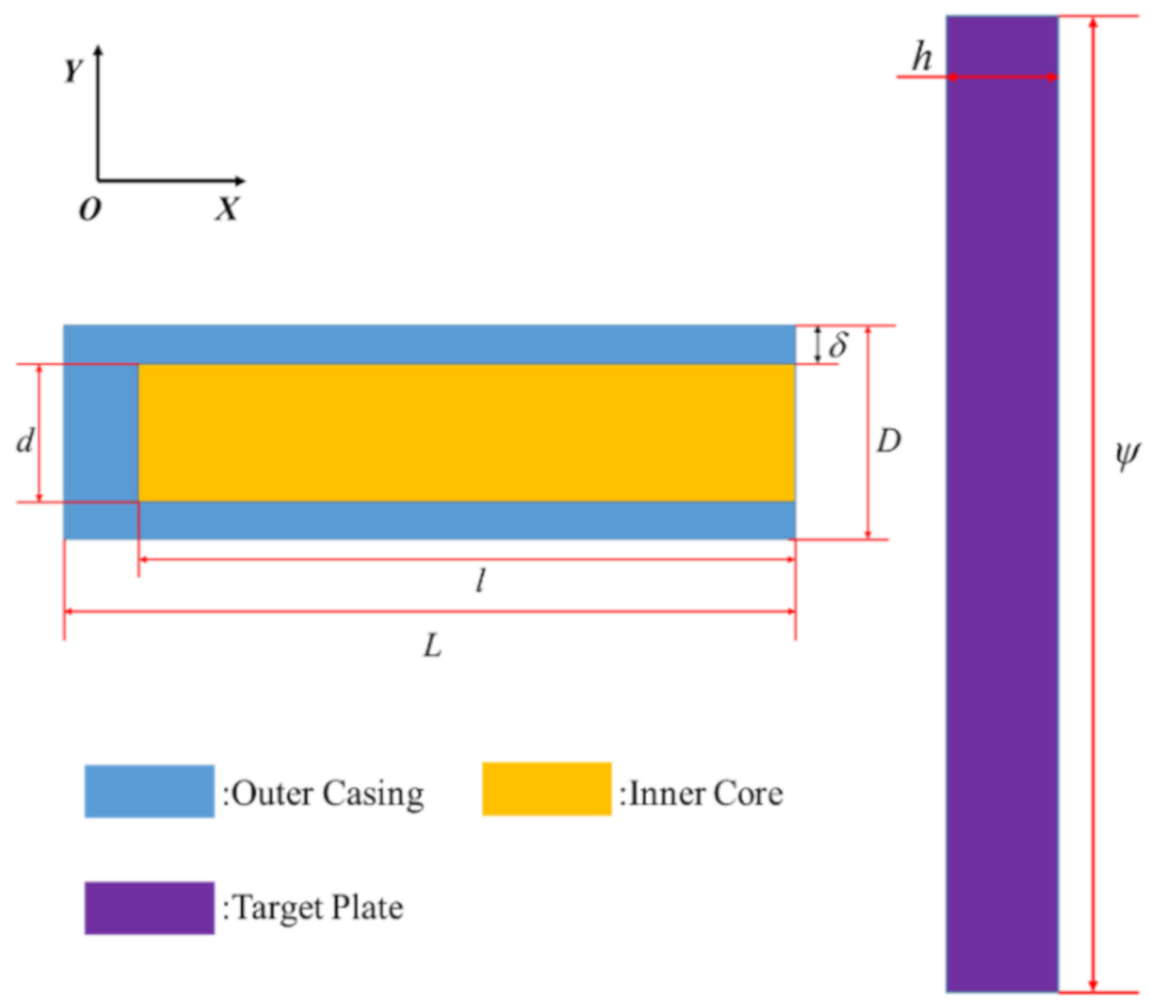

Figure 6. Schematic diagram of the PELE projectile and target plate.

According to the previous basic assumption, the projectile will propagate shock waves to the projectile and the target plate respectively when the impact occurs. The propagation velocity of shock wave in the outer casing and the inner core of PELE projectile is $C_{j}$ and $C_{f}$ respectively, and the directions are opposite to the movement direction of projectile. The propagation velocity of shock wave in the target plate is $C_{t}$, the direction is consistent with the movement direction of projectile. Under the impact action, the contact stress of the outer casing and inner core in the impact end is $\sigma_{j}$ and $\sigma_{f}$, and the contact stress will cause the contact interfaces between the two part and the target plate to obtain velocity, which are denoted by $u_{j}$ and $u_{f}$. Therefore, according to the shock wave theory and momentum conservation relationship between the PELE projectile and target plate during impact process, the following relationship can be obtained:

$$
\left\{\begin{aligned}
\sigma_{j} & =u_{0} \frac{\rho_{j} C_{j} \rho_{t} C_{t}}{\rho_{j} C_{j}+\rho_{t} C_{t}} \\
\sigma_{f} & =u_{0} \frac{\rho_{f} C_{f} \rho_{t} C_{t}}{\rho_{f} C_{f}+\rho_{t} C_{t}}
\end{aligned}\right.
$$




$$
\left\{\begin{array}{l}
u_{j}=u_{0} \frac{\rho_{j} C_{j}}{\rho_{j} C_{j}+\rho_{t} C_{t}} \\
u_{f}=u_{0} \frac{\rho_{f} C_{f}}{\rho_{f} C_{f}+\rho_{t} C_{t}}
\end{array}\right.
$$

When the projectile impacts the target plate, it will produce strain in the axial direction due to the axial pressure, that is, $\varepsilon_{x}=\partial s_{x} / \partial x=-\sigma_{x}(x, t) / E$. Therefore, the radial deformation will inevitably be caused by Poisson effect, and the relational expression of radial strain is as follows:

$$
\left\{\begin{aligned}
\varepsilon_{y} & =\partial s_{y} / \partial y=-\mu \varepsilon_{x}(x, t) \\
\varepsilon_{z} & =\partial s_{z} / \partial z=-\mu \varepsilon_{x}(x, t)
\end{aligned}\right.
$$

where $s_{x}, s_{y}$ and $s_{z}$ are respectively expressed as displacement components in the $x$-axis, $y$-axis and $z$-axis directions. By integrating the Formula (3), the radial displacements $s_{y}$ and $s_{z}$ can be obtained.

$$
\left\{\begin{aligned}
s_{y} & =-\mu y \varepsilon_{x}(x, t)=-\mu y \frac{\partial s_{x}(x, t)}{\partial x} \\
s_{z} & =-\mu z \varepsilon_{x}(x, t)=-\mu z \frac{\partial s_{x}(x, t)}{\partial x}
\end{aligned}\right.
$$

Based on the above radial displacement relationship, the derivative of time $t$ can be used to obtain the radial particle velocity $u_{y}$ and $u_{z}$ as follows:

$$
\left\{\begin{array}{l}
u_{y}=\frac{\partial s_{y}}{\partial t}=-\mu y \frac{\partial \varepsilon_{x}}{\partial t}=-\mu y \frac{\partial u_{x}}{\partial x} \\
u_{z}=\frac{\partial s_{z}}{\partial t}=-\mu z \frac{\partial \varepsilon_{x}}{\partial t}=-\mu z \frac{\partial u_{x}}{\partial x}
\end{array}\right.
$$

According to the kinetic energy relationship, the average radial kinetic energy $E_{1}$ in a unit volume can be obtained as follows:

$$
E_{1}=\frac{1}{\mathrm{~A}_{0} \mathrm{~d} x} \int_{\mathrm{A}_{0}} \rho\left(u_{y}{ }^{2}+u_{z}{ }^{2}\right) \mathrm{d} x \mathrm{~d} y \mathrm{~d} z=\frac{1}{2} \rho \mu^{2} r_{g}^{2}\left(\partial \varepsilon_{x} / \partial t\right)^{2}
$$

where $A_{0}$ is the cross-sectional area, $\rho$ is the density, $\mu$ is the Poisson's ratio, $\varepsilon$ is the strain, $r_{g}$ is the rotating radius of the cross section to the projectile axis ( $x$-axis), and its general expression is $r_{g}=\frac{1}{\mathrm{~A}_{0}} \int_{\mathrm{A}_{0}}\left(y^{2}+z^{2}\right) \mathrm{d} y \mathrm{~d} z$. For the hollow tube-shaped interface, the rotating radius can also be expressed as $r_{g}^{2}=\frac{D \sqrt{1+\gamma^{2}}}{4}$.

According to the definition of strain, the axial strain $\varepsilon_{x}$ can also be expressed as $\varepsilon_{x}=\mathrm{d} l / L$. Therefore, the expression of axial strain rate can be written as:

$$
\dot{\varepsilon}=\frac{\mathrm{d} l}{L \mathrm{~d} t}
$$

Based on the description in the Tate model [22], there exists the following relationship $\mathrm{d} l / \mathrm{d} t=$ $u_{0}-u_{j}$. Therefore, the axial strain rate can be rewritten as:

$$
\dot{\varepsilon}=\frac{\mathrm{d} l}{L \mathrm{~d} t}=\frac{u_{0}-u_{j}}{L}
$$

Therefore, the radial kinetic energy of the unit volume outer casing corresponding to the fragments can be rewritten as:

$$
E_{1}=\rho_{j} u_{r 1}^{2} / 2
$$

By combining the above formulas, the radial scattering velocity component of fragments corresponding to the kinetic energy of outer casing can be calculated as follows:

$$
u_{r 1}=\frac{u_{0} \mu_{j} \sqrt{1+\gamma^{2}}}{4 \zeta\left(1+\rho_{j} C_{j} / \rho_{t} C_{t}\right)}
$$


Due to the axial compression of inner core in the process of projectile-target action,, the Poisson effect makes the compression energy of inner core convert into the radial force on the outer casing. Formula (1) has given the contact stress $\sigma_{f}$ at the interface between the inner core and the target plate at the moment of projectile-target action, so the radial stress acting on the inner wall of the outer casing can be obtained:

$$
\sigma_{f-j}=\frac{1-\mu_{f}}{\mu_{f}} \sigma_{f}=u_{0} \frac{1-\mu_{f}}{\mu_{f}} \frac{\rho_{f} C_{f} \rho_{t} C_{t}}{\rho_{f} C_{f}+\rho_{t} C_{t}}
$$

Under the condition of high-speed impact, the inner core material approximately presents a fluid state based on the basic assumption (1), so $\mu_{f}$ can be approximately taken as 0.5 . Therefore, there is the following relation according to the momentum theorem:

$$
\sigma_{f-j} \mathrm{~A} t=m u_{r 2}
$$

where $A$ represents the contact area between the inner core and the outer casing, $m$ represents the mass corresponding to the unit length outer casing, which can be expressed as $m=\pi \rho_{j} D^{2}\left(1-\zeta^{2}\right) / 4 . t$ is the action time of force, corresponding to the back and forth time of the stress wave in the target plate, which can be expressed as $t=2 h / C_{t}$.

By combining the Formulas (11) and (12), we can obtain the expression of $u_{r 2}$ as follows:

$$
u_{r 2}=u_{0} \frac{8 h \zeta}{D\left(1-\zeta^{2}\right)} \frac{1}{\left(\rho_{j} / \rho_{t}\right)\left(1+\rho_{t} C_{t} / \rho_{f} C_{f}\right)}
$$

At the same time, the unit volume energy $E_{2}$ corresponding to $u_{r 2}$ can be reversed as:

$$
E_{2}=\rho_{j} u_{r 2}^{2} / 2
$$

The radial motion of fragments is not only attributed to the above two energies, but also includes the chemical energy released by the reactive core material. The mechanism of this part of energy acting on the outer casing can be analyzed with reference to the fragment scattering mechanism of the cylindrical fragmentation warhead. For the cylindrical fragmentation warhead, according to the conservation of energy after explosion [23], there are the following relationships:

$$
\left\{\begin{array}{c}
C E=\frac{1}{2} M v_{0}^{2}+\frac{1}{4} C v_{0}^{2} \\
C E=\frac{1}{2}(M+C / 2) v_{0}^{2} \\
v_{0}=\sqrt{2 E} \sqrt{\frac{C}{M+C / 2}}
\end{array}\right.
$$

where $C$ is the mass of explosive, $E$ is the total energy released after the explosion of the unit mass explosive charge, $M$ is the mass of casing, $v_{0}$ is the scattering velocity of fragments.

The energy released by the reaction of reactive core material per unit mass is $Q$. Therefore, according to the energy conservation principle, the following relational expression can be obtained:

$$
Q \rho_{f}=\frac{\pi\left(D^{2}-d^{2}\right)}{8} \rho_{j} u_{r 3}^{2}
$$

By solving the Formulas (15) and (16), we can obtain the following expression:

$$
u_{r 3}=\sqrt{\frac{8 Q \rho_{f}}{\pi \rho_{j}\left(D^{2}-d^{2}\right)}}
$$

At the same time, the unit volume energy $E_{3}$ corresponding to $u_{r 3}$ can also be reversed as:

$$
E_{3}=\rho_{j} u_{r 3}^{2} / 2
$$


Based on the above analysis, the contribution components of three energy sources to the radial scattering velocity of fragments have been obtained. Note that the total radial scattering velocity of fragments generated after the projectile perforates the target plate is $u_{r}$, then there are the following relationships:

$$
\begin{aligned}
& E_{1}+E_{2}+E_{3}=\frac{1}{2} \rho_{j} u_{r}^{2} \\
& u_{r}=\sqrt{u_{r 1}^{2}+u_{r 2}^{2}+u_{r 3}^{2}}
\end{aligned}
$$

At this point, the theoretical model of radial scattering velocity of fragments of the reactive core PELE projectile has been established.

\section{Numerical Simulation Verification of the Theoretical Model}

As a special type of penetrator, the significant difference between the reactive core PELE projectile and the traditional PELE projectile is that it has significant fragmentation effect. Since it is difficult to accurately measure the radial scattering velocity of fragments after the reactive core PELE projectile penetrates the target through experiments, it is necessary to carry out research by means of numerical simulation. Therefore, this paper will set up a number of different working conditions to verify the theoretical model.

\subsection{Finite Element Model}

In view of our team has carried out a lot of numerical simulation research on PELE projectiles [24-26], and we can well simulate the penetration and damage of PELE projectiles to the target plate. Therefore, this paper will still use AUTODYN (Century Dynamics, Fort Worth, TX, USA) nonlinear dynamics software to simulate the penetration process of the reactive core PELE projectile.

According to the previous model simplification, it can be known that the entire numerical simulation model mainly includes three parts: outer casing, reactive inner core and target plate. In the modeling process, unstructured hexahedral meshes are used in both the projectile and the target plate, and the average size of mesh is $0.25 \mathrm{~mm}$. First, the HyperMesh software (Altair Engineering, Detroit, MI, USA) is used for modeling, and then the finite element model is imported into AUTODYN for solution. In order to reduce the total number of meshes to improve the calculation efficiency, the meshes of target plate adopt the variable-step size. The mesh density in the central area of the target plate is about twice the projectile diameter, and the minimum mesh size is $0.25 \mathrm{~mm}$. The reactive core material is modeled by SPH particles with particle size of $0.5 \mathrm{~mm}$. Since the rotation of the projectile is not considered in this paper, only the vertical impact of the projectile on the target plate is considered, so the model can be simplified by $1 / 4$ model. For the reactive core PELE projectile, the reaction of the reactive core material is usually incomplete, so the reactive core material is modeled in two parts. The equation of state used in the two parts are also different, that is, the part near the impact end is defined as the reaction part, and the part near the projectile rear is defined as the unreacted part. The proportion of the two parts of the reactive core material is controlled by the reaction percentage $\beta=x_{c} / l$. The structural parameters of the reactive core PELE projectile designed in this paper are shown in Table 1. Taking the working condition of the reaction percentage $\beta=50 \%$ as an example, its finite element model is shown in Figure 7. In Figure 7, blue represents the target plate, green represents the outer casing, yellow represents the reactive material that did not participate in the reaction, and purple represents the reactive material that participated in the reaction. The components represented by colors in all the numerical simulation conditions below are consistent with Figure 7 . 
Table 1. Structural parameters of the reactive core pele projectile.

\begin{tabular}{cccccc}
\hline $\begin{array}{c}\text { Projectile } \\
\text { Length } L(\mathbf{m m})\end{array}$ & $\begin{array}{c}\text { Outer Casing } \\
\text { Diameter } D \\
(\mathbf{m m})\end{array}$ & $\begin{array}{c}\text { Inner Core } \\
\text { Diameter } d \\
(\mathbf{m m})\end{array}$ & $\begin{array}{c}\text { Outer Casing } \\
\text { Thickness } \sigma \\
(\mathbf{m m})\end{array}$ & $\begin{array}{c}\text { Inner core } \\
\text { Length } l(\mathbf{m m})\end{array}$ & $\begin{array}{c}\text { Target Plate } \\
\text { Thickness } h \\
(\mathbf{m m})\end{array}$ \\
\hline 70 & 28 & 22 & 3 & 64 & $\begin{array}{c}\text { Target Plate } \\
\text { Length } \psi(\mathbf{m m})\end{array}$ \\
\hline
\end{tabular}

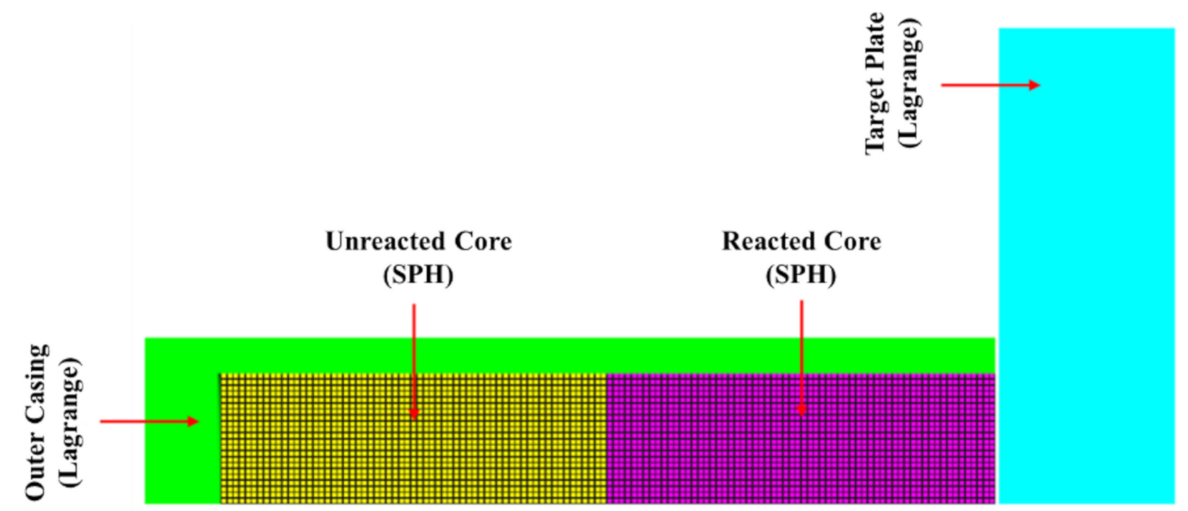

Figure 7. Finite element model of the reactive core PELE projectile ( $\beta=50 \%)$.

\subsection{Material Model}

AUTODYN software mainly includes four commonly used algorithms: Lagrange, Euler, ALE, SPH [27]. Lagrange algorithm can usually be used to simulate the mechanical response of solid materials, and the calculation speed is fast. However, it cannot solve the large deformation problem well, and the calculation accuracy and efficiency will be greatly reduced due to the mesh distortion. The main feature of the Euler algorithm is that the spatial position coordinates of the Euler mesh are fixed, which allows material to flow in and out of the mesh freely, without involving mesh distortion. Therefore, the Euler algorithm is usually used to describe the mechanical behavior of gases and liquids. The ALE algorithm combines the advantages of Lagrange algorithm and Euler algorithm, that is, the mesh corresponding to the ALE algorithm can be fixed or flow with matter. Therefore, the ALE algorithm is usually used to solve the coupling problem of various fluid structures. The SPH algorithm is a meshless numerical simulation method, its basic idea is to discretize the matter in the flow field into many smooth particles with mass, energy and velocity. Therefore, the SPH algorithm is mainly used to solve large deformation problems, such as breaking and disintegration of various structures. Based on the above detailed analysis of the four algorithms, combined with the characteristics of each part of the material, the algorithm used in each part of the numerical simulation in this paper can be determined, that is, the outer casing and the target plate use the Lagrange algorithm, and the reactive core uses the $\mathrm{SPH}$ algorithm.

The material of outer casing is tungsten alloy, and the principal stress/strain failure model is adopted, that is, when the tensile principal strain reaches $\varepsilon_{\mathrm{T}}$ or the tensile principal stress reaches $\sigma_{\mathrm{T}}$, the material is considered invalid. The material of target plate is steel, and the plastic strain failure model is used. It is believed that when the plastic strain reaches a certain value, the material will fail. The main component of the reactive material in this paper is selected as PTFE/Al, which is mainly divided into two parts: the reacted part and the unreacted part. According to the reaction characteristics of the reactive material, the equation of state of the reacted part adopts the Powder Burn equation, while the equation of state of the unreacted part adopts the Shock equation. In addition, regardless of whether the reactive material uses the Powder Burn equation of state or the Shock equation of state, they will be used in conjunction with the Johnson-Cook strength model and the principal stress failure model. Based on the above analysis, the material model of each part of the entire finite element model can be obtained as shown in Table 2. 
Table 2. Material model of each part.

\begin{tabular}{ccccc}
\hline Part & Material & EOS & Strength Model & Failure Model \\
\hline Outer casing & Tungsten & Shock & Johnson-Cook & Principal \\
stress/strain \\
Inner core (Reacted) & PTFE/Al & Powder Burn & Johnson-Cook & Principal stress \\
Inner core (Unreacted) & Steel & Shock & Johnson-Cook & Principal stress \\
Target plate & Shock & von Mises & Principal strain \\
\hline
\end{tabular}

\subsection{Simulation Condition}

The main variable in this paper is the impact velocity of the reactive core PELE projectile. According to the theoretical analysis of shock wave, it can be known that different impact velocity will cause different internal pressures of the inner core. The reaction degree of the reactive core material is positively correlated with the initial excitation energy, which means that the higher the impact velocity of the projectile is, the higher the reaction percentage of the reactive core material is. To this end, four different impact velocities $(800 \mathrm{~m} / \mathrm{s}, 1000 \mathrm{~m} / \mathrm{s}, 1200 \mathrm{~m} / \mathrm{s}$, and $1400 \mathrm{~m} / \mathrm{s})$ are designed in this paper, and the thickness of the steel target plate is $15 \mathrm{~mm}$. The pressure distribution in the inner core is approximately exponentially attenuated when the PELE projectile impacts the target plate. Therefore, the reactive core material reaction percentages $\beta$ corresponding to the four impact velocities $(800 \mathrm{~m} / \mathrm{s}$, $1000 \mathrm{~m} / \mathrm{s}, 1200 \mathrm{~m} / \mathrm{s}$, and $1400 \mathrm{~m} / \mathrm{s})$ can be calculated as $(47 \%, 50 \%, 54 \%$ and $57 \%)$, the length of the reacted and unreacted part corresponding to different reaction percentage $\beta$ can also be obtained. In summary, the numerical simulation conditions can be obtained as shown in Table 3, and the finite element model of the projectile corresponding to each condition is shown in Figure 8.

Table 3. Simulation condition.

\begin{tabular}{ccccc}
\hline $\begin{array}{c}\text { Condition } \\
\text { Number }\end{array}$ & $\begin{array}{c}\text { Impact Velocity } \boldsymbol{v}_{\mathbf{0}} \\
(\mathbf{m} / \mathbf{s})\end{array}$ & $\begin{array}{c}\text { Reaction } \\
\text { Percentage } \boldsymbol{\beta}(\boldsymbol{\%})\end{array}$ & $\begin{array}{c}\text { Length of Reacted Core } \\
\boldsymbol{l}_{\mathbf{1}}(\mathbf{m m})\end{array}$ & $\begin{array}{c}\text { Length of Unreacted Core } \\
\boldsymbol{l}_{\mathbf{2}}(\mathbf{m m})\end{array}$ \\
\hline$\# 1$ & 800 & 47 & 30 & 34 \\
$\# 2$ & 1000 & 50 & 32 & 32 \\
$\# 3$ & 1200 & 54 & 34.5 & 29.5 \\
$\# 4$ & 1400 & 57 & 36.5 & 27.5 \\
\hline
\end{tabular}

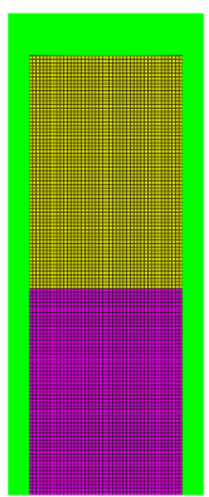

(a)

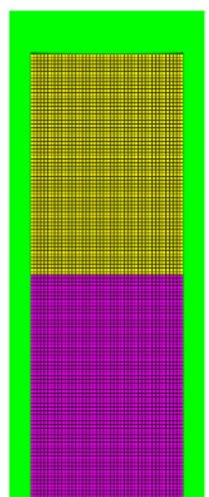

(b)

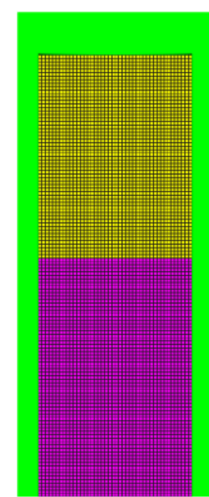

(c)

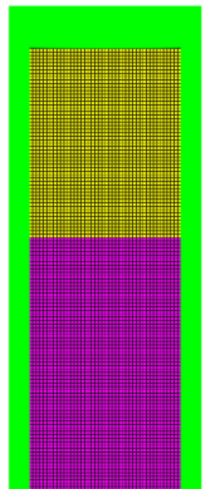

(d)

Figure 8. Finite element model of projectile corresponding to different impact velocities. (a) $v=800 \mathrm{~m} / \mathrm{s}$ $(\beta=47 \%),(\mathbf{b}) v=1000 \mathrm{~m} / \mathrm{s}(\beta=50 \%),(\mathbf{c}) v=1200 \mathrm{~m} / \mathrm{s}(\beta=54 \%),(d) v=1400 \mathrm{~m} / \mathrm{s}(\beta=57 \%)$.

\subsection{Analysis of the Numerical Simulation Results}

In Section 4.3, the theoretical model of radial scattering velocity of fragments of the reactive core PELE projectile has been established. In order to verify the rationality of the theoretical model, the numerical simulation results and theoretical calculation results will be compared and analyzed in this section. As can be seen from the foregoing, a series of simulation conditions have been designed in this paper. The spatial distribution of fragments after the reactive core PELE projectile perforates the 
target corresponding to each condition is shown in Figure 9. Because the reactive core material adopts the SPH algorithm, the inner core is composed of a large number of smooth particles, which will cause the fragments to be covered by the SPH particles when flying. Therefore, in order to see the flying state of fragments, two forms of display are used during the display in Figure 9, that is, show the SPH particles and not show the SPH particles.

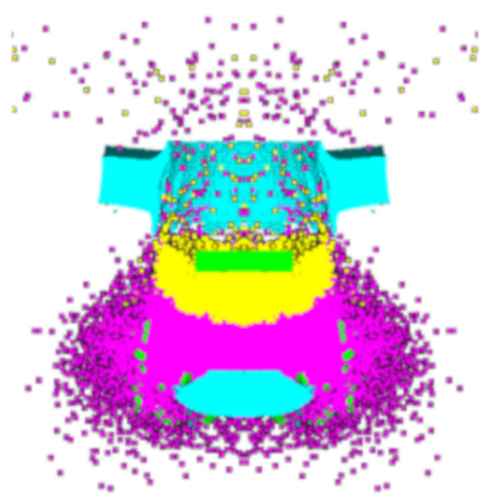

(a)

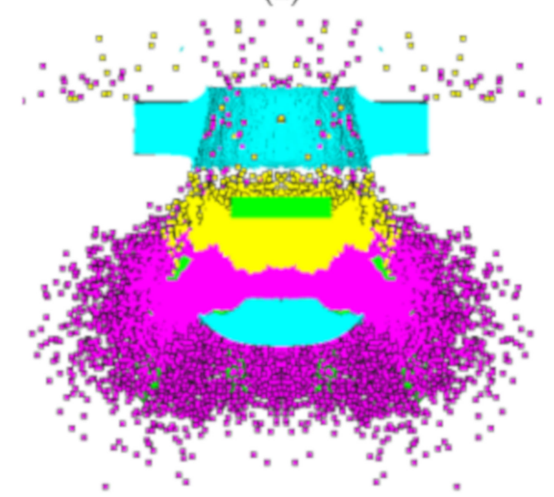

(c)

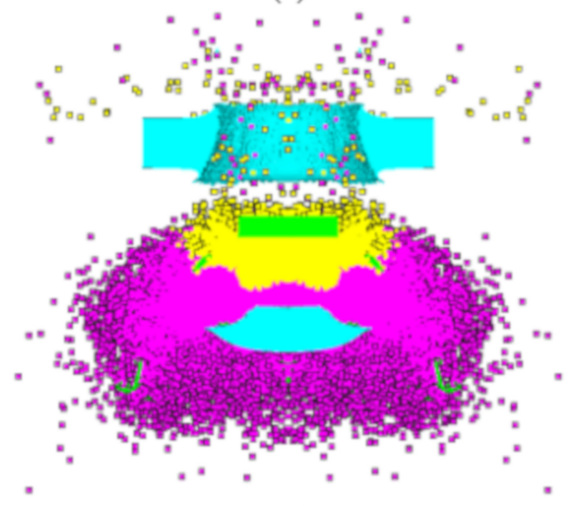

(e)
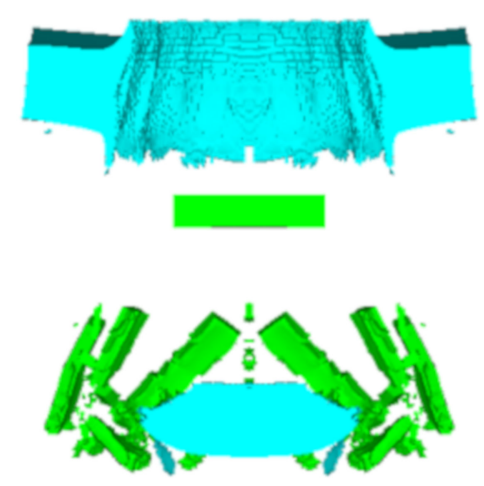

(b)

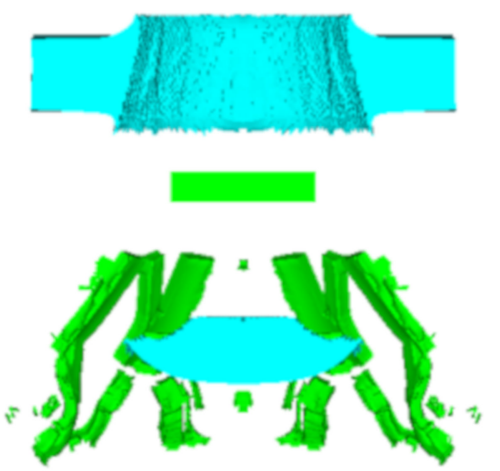

(d)

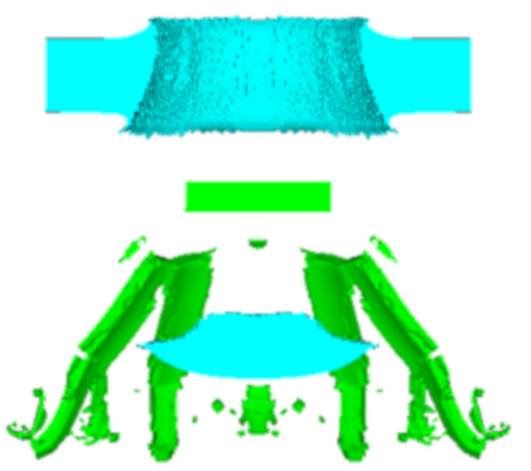

(f)

Figure 9. Cont. 


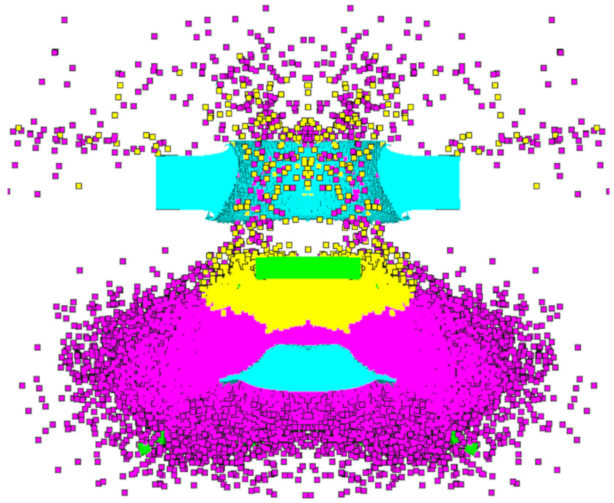

(g)

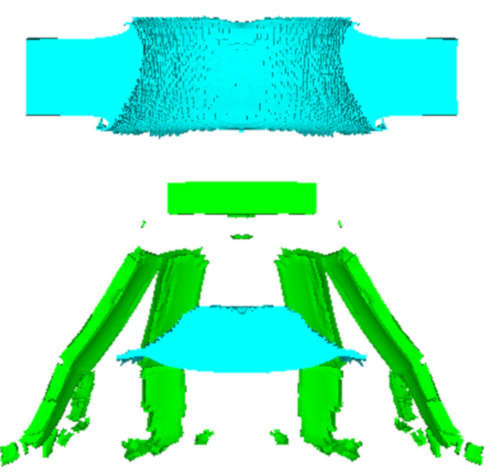

(h)

Figure 9. Spatial distribution of fragments after the reactive core PELE projectile perforates the target corresponding to each condition: (a) $v=800 \mathrm{~m} / \mathrm{s}$ (show the SPH particles); (b) $v=800 \mathrm{~m} / \mathrm{s}$ (not show the $\mathrm{SPH}$ particles); (c) $v=1000 \mathrm{~m} / \mathrm{s}$ (show the SPH particles); (d) $v=1000 \mathrm{~m} / \mathrm{s}$ (not show the SPH particles); (e) $v=1200 \mathrm{~m} / \mathrm{s}$ (show the SPH particles); (f) $v=1200 \mathrm{~m} / \mathrm{s}$ (not show the SPH particles); (g) $v=1400 \mathrm{~m} / \mathrm{s}$ (show the SPH particles); (h) $v=1400 \mathrm{~m} / \mathrm{s}$ (not show the SPH particles).

The simulation results of the radial scattering velocity of fragments after the reactive core PELE projectile perforates the target plate can be obtained, as shown in Table 4. Similarly, the theoretical results of the radial scattering velocity of fragments can also be obtained by substituting the various known conditions into the theoretical model, as shown in Table 4. In order to visually compare and analyze the difference between the simulation results and the theoretical results, the two are displayed in the form of histogram, as shown in Figure 10.

Table 4. Simulation and theoretical results of the radial scattering velocity of fragments of the reactive core pele projectile.

\begin{tabular}{cccc}
\hline \multirow{2}{*}{ Impact Velocity $\boldsymbol{v}_{\mathbf{0}}(\mathbf{m} / \mathbf{s})$} & \multicolumn{2}{c}{ Radial Scattering Velocity of Fragments $\boldsymbol{u}_{\mathbf{r}}(\mathbf{m} / \mathbf{s})$} & \multirow{2}{*}{ Error $\boldsymbol{\delta}(\%)$} \\
\cline { 2 - 3 } & Simulation Result & Theoretical Result & \\
\hline 800 & 177 & 189 & 6.3 \\
1000 & 264 & 281 & 6.0 \\
1200 & 426 & 445 & 4.3 \\
1400 & 548 & 570 & 3.9 \\
\hline
\end{tabular}

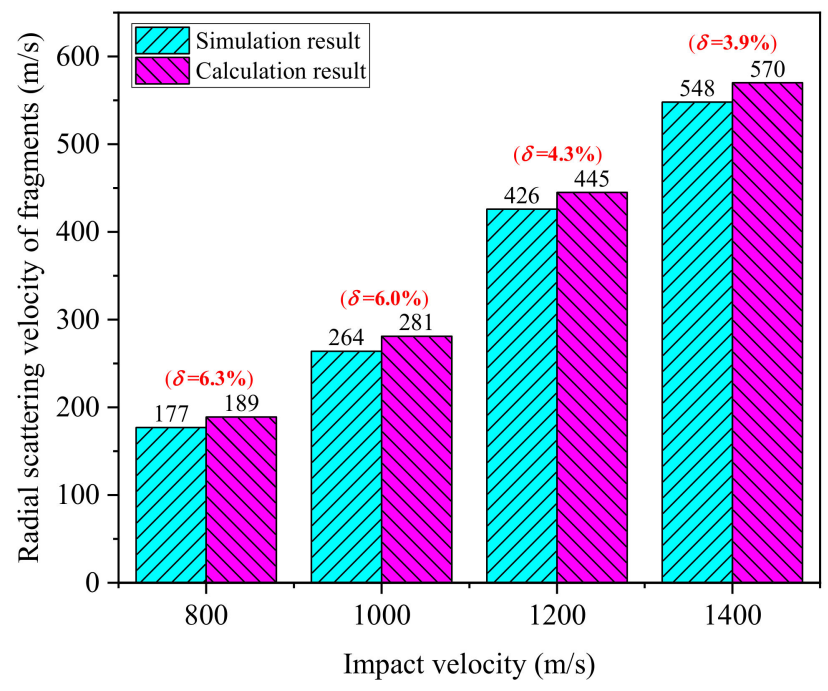

Figure 10. Histogram of the simulation results and theoretical results. 
Based on the above comparative analysis, the following conclusions can be obtained from Table 4 and Figure 10:

(1) The radial scattering velocity of fragments increases with the increase of impact velocity. Because the reaction percentage $\beta$ of reactive core material increases with the increase of impact velocity, which means that more chemical energy is released from the reactive core material, and more energy is converted into the radial scattering velocity of fragments.

(2) The calculation results obtained from the theoretical model are slightly higher than the numerical simulation results. Because there are a lot of simplifications when constructing the theoretical model, ignoring a lot of energy dissipation, in other words, most of the energy is used to convert to the kinetic energy of fragments.

(3) The error between the numerical simulation results and calculation results is within $6.5 \%$, and the error between them decreases with the increase of impact velocity. Because with the increase of impact velocity, the total energy used to convert into the kinetic energy of fragments increases, which means that the proportion of dissipated energy to total energy decreases, so the theoretical results will be closer to the numerical simulation results.

Based on the above analysis, it can be considered that the theoretical model of radial scattering velocity of fragments of the reactive core PELE projectile established in this paper is scientific and reasonable, which has important theoretical reference value and can provide guidance for engineering design. In the next step, we also consider trying to carry out some experiments to further verify the theoretical model. The experiments may be carried out mainly by means of the combination of $\mathrm{X}$-ray photography and high-speed photography to complete the analysis of the radial scattering velocity of fragments. In the experiments, the experiment parameters that may be mainly concerned include the radial displacement of fragments, the radial movement time of fragments, and the penetration time of fragments, etc.

\section{Conclusions}

The penetration damage mechanism of the reactive core PELE projectile is different from the traditional PELE projectile. Firstly, the structure of PELE projectile was simplified, and the penetration process of the reactive core PELE projectile was analyzed. Secondly, the energy source of radial scattering velocity of fragments after the reactive core PELE projectile perforates the target was analyzed. Based on the basic assumptions, the theoretical model of radial scattering velocity of fragments of the reactive core PELE projectile was established. Finally, numerical simulations were carried out to verify the scientificity and rationality of the theoretical model. Based on the comprehensive analysis of the full text, the following main conclusions can be obtained:

(1) There is a significant difference between the penetration damage mechanism of the reactive core PELE projectile and that of the tarditional PELE projectile, which is mainly reflected in the active release of chemical energy by the reactive materials in the penetration process, so the reactive core PELE projectile has stronger fragmentation effect.

(2) The energy source of the radial scattering velocity of fragments can be summarized into the following three parts: the axial kinetic energy of outer casing, the radial compression potential energy generated by the inner core to the outer casing, and the chemical energy released by the reactive core material. According to the theory of shock wave and the principle of energy conservation, the three energy sources are analyzed respectively, and the theoretical expression of the radial scattering velocity of fragments is obtained.

(3) The theoretical model established in this paper is verified by carrying out a number of numerical simulations. The comparison results show that the radial scattering velocities of fragments obtained by the two methods are in good agreement, and the error is within the acceptable range. It can be considered that the theoretical model of radial scattering velocity of fragments of the reactive core PELE projectile has a certain theoretical reference value. 
In summary, the theoretical model of radial scattering velocity of fragments of the reactive core PELE projectile established in this paper is scientific and reasonable, which has significant theoretical reference value and can provide guidance for engineering design.

Author Contributions: Conceptualization, L.D. and W.T.; methodology, L.D.; software, L.D. and J.Z.; validation, J.Z., X.R. and X.X.; formal analysis, J.Z. and X.X.; resources, X.R.; data curation, X.X. and Y.Z.; writing-Original draft preparation, L.D.; writing-Review and editing, W.T.; supervision, W.T. and Y.Z.; project administration, W.T.; funding acquisition, X.R. and W.T. All authors have read and agreed to the published version of the manuscript.

Funding: This research was funded by the National Natural Science Foundation of China (grant No. 11002162 and 11072262).

Conflicts of Interest: The authors declare no conflict of interest.

\section{References}

1. Kesberg, G.; Schirm, V.; Kerk, S. PELE: The future ammunition concept. In Proceedings of the 21st International Symposium on Ballistics (ISB'21), Adelaide, Australia, 19-23 April 2004.

2. Paulus, G.; Chanteret, P.Y.; Wollmann, E. PELE: A new penetrator concept for generating lateral effects. In Proceedings of the 21st International Symposium on Ballistics (ISB'21), Adelaide, Australia, 19-23 April 2004.

3. Paulus, G.; Schirm, V. Impact behavior of PELE projectiles perforating thin target plates. Int. J. Impact Eng. 2006, 33, 566-579. [CrossRef]

4. Rheinmetall Waffe Munition. 105/120/125 mm PELE Firing Results. Available online: https://ndiastorage. blob.core.usgovcloudapi.net/ndia/2005/garm/wednesday/borngen.pdf (accessed on 21 June 2020).

5. Gloude, D. Capabilities of Penetrator with Enhanced Lateral Efficiency. Available online: https://ndiastorage. blob.core.usgovcloudapi.net/ndia/2007/gun_missile/GMTueAM1/GloudePresentation.pdf (accessed on 21 June 2020).

6. Mock, W.; Drotar, J.T. Effect of aluminum particle size on the impact initiation of pressed PTFE/Al composite rods. AIP Conf. Proc. 2007, 955, 971-974.

7. Yang, S.; Xu, S.; Zhang, T. Preparation and performance of PTFE/Al reactive materials. J. Natl. Univ. Def. Technol. 2008, 30, 39-42.

8. Huang, H.; Huang, H.; Yang, S.; Yang, P.; Zhang, T.; Xi, Y.; Lu, X. Preliminary research on damage enhanced fragment. Chin. J. Energ. Mater. 2007, 15, 566-569.

9. Ran, X.; Ding, L.; Zhou, J.; Tang, W. Research on the Energy Release Characteristics of Six Kinds of Reactive Materials. Materials 2019, 12, 3940. [CrossRef] [PubMed]

10. Vasant, S.; Waldorf, M. Process for Making Polytetrafluoroethylene-Aluminum Composite and Product Made. U.S. Patent 6547993B1, 15 April 2003.

11. Ding, L.; Zhou, J.; Tang, W.; Ran, X.; Hu, Y. Impact Energy Release Characteristics of PTFE/Al/CuO Reactive Materials Measured by a New Energy Release Testing Device. Polymers 2019, 11, 149. [CrossRef] [PubMed]

12. Zhou, J.; Ding, L.; Tang, W.; Ran, X. Experimental Study of Mechanical Properties and Impact-Induced Reaction Characteristics of PTFE/Al/CuO Reactive Materials. Materials 2020, 13, 66. [CrossRef] [PubMed]

13. Thadhani, N. Shock-induced and shock-assisted solid-state chemical reactions in powder mixtures. J. Appl. Phys. 1994, 76, 2129-2138. [CrossRef]

14. Song, I.; Thadhani, N. Shock-induced chemical reactions and synthesis of nickel aluminides. Metall. Mater. Trans. A 1992, 23, 41-48. [CrossRef]

15. Verreault, J. Analytical and numerical description of the PELE fragmentation upon impact with thin target plates. Int. J. Impact Eng. 2015, 76, 196-206. [CrossRef]

16. Verreault, J.; Hinsberg, N.; Abadjieva, E. PELE fragmentation dynamics. In Proceedings of the 27th International Symposium on Ballistics (ISB'27), Freiburg, Germany, 22-26 April 2013.

17. Verreault, J. Modeling of the PELE fragmentation dynamics. In Proceedings of the 18th Biennial International Conference of the APS Topical Group on Shock Compression of Condensed Matter held in conjunction with the 24th Biennial International Conference of the International Association for the Advancement of High Pressure Science and Technology, Seattle, WA, USA, 7-12 July 2013.

18. Zhu, J.; Zhao, G.; Du, Z.; Wang, X. Mechanism of PELE projectiles perpendicularly impacting on thin target plates. Explos. Shock Waves 2009, 29, 281-288. 
19. Du, Z.; Song, L.; Zhong, K.; Wang, F. Influence of the ratio of inner to outer diameter on penetrator with enhanced lateral efficiency. J. Comput. Theor. Nanosci. 2011, 4, 1525-1528. [CrossRef]

20. Fan, Z.; Ran, X.; Tang, W.; Ke, Y.; Li, Z. The model to calculate the radial velocities of fragments after PELE perforating a thin plate. Int. J. Impact Eng. 2016, 95, 12-16. [CrossRef]

21. Fan, Z.; Ran, X.; Tang, W.; Yu, G.; Chen, W.; Ren, C. Calculation method and influencing factors of the fragmental radial velocities of PELE after penetrating thin target. Explos. Shock Waves 2017, 37, 621-628.

22. Zhao, G. Engineering Mechanics of Armor Piercing; Ordnance Industry Press: Beijing, China, 1992.

23. Beijing Institute of Technology «explosion and its effects» Compile Group. Explosion and Its Effects; National Defense Industry Press: Beijing, China, 1979.

24. Ding, L.; Zhou, J.; Tang, W.; Ran, X.; Cheng, Y. Research on the crushing process of PELE casing material based on the crack-softening algorithm and stochastic failure algorithm. Materials 2018, 11, 1561. [CrossRef] [PubMed]

25. Ding, L.; Zhou, J.; Tang, W.; Ran, X.; Cheng, Y. Damage characteristics of PELE projectile with gradient density inner core material. Materials 2018, 11, 2389. [CrossRef] [PubMed]

26. Ding, L.; Zhou, J.; Tang, W.; Ran, X. Damage characteristics analysis of the truncated Cone-Shaped PELE projectile. Symmetry 2019, 11, 1025. [CrossRef]

27. Century Dynamics Inc. Interactive Non-Linear Dynamic Analysis Software AUTODYN User Manual; Century Dynamics Inc.: Oakland, CA, USA, 2003.

(C) 2020 by the authors. Licensee MDPI, Basel, Switzerland. This article is an open access article distributed under the terms and conditions of the Creative Commons Attribution (CC BY) license (http://creativecommons.org/licenses/by/4.0/). 